EPJ Web of Conferences 22, 00013 (2012)

DOI: $10.1051 /$ epjconf/20122200013

(C) Owned by the authors, published by EDP Sciences, 2012

\title{
An introduction to the use of representation analysis for studying magnetoelectrics and multiferroics
}

\author{
L.C. Chapon ${ }^{1,2}$ \\ ${ }^{1}$ ISIS facility, STFC Rutherford Appleton Laboratory, Chilton, Didcot, Oxfordshire OX11 OQX, \\ UK \\ ${ }^{2}$ Institut Laue-Langevin, BP. 156, 6 rue Jules Horowitz, 38042 Grenoble Cedex 9, France
}

\begin{abstract}
This lecture is an introduction to the theory of representations applied to the study of magnetoelectric and multiferroic materials. It is intended for students or newcomers in the field and explains the key concepts required to understand phenomenologically the coupling between magnetic phase transitions in crystals and dielectric properties. Symmetry properties of some prototypal magnetoelectrics and multiferroics are analysed, including the treatment of incommensurate spin-driven ferroelectrics. It is deliberately written with a minimal use of mathematical formulation or a strict group theoretical approach.
\end{abstract}

\section{INTRODUCTION}

In the last ten years, there has been a regain of interest for magnetoelectrics and magnetic-ferroelectrics, often referred to as multiferroics. The term multiferroic, first used by Shmidt [1], describes systems in which at least two out of the three ferroic orders - ferromagnetism, ferroelasticity and ferroelectricity - coexist. By extension, this terminology has been employed to describe systems that combine ferroelectric (FE) order and antiferromagnetic (AFM) order, whether the transition paraelectric (PE) $\rightarrow$ FE and paramagnetic $(\mathrm{PM}) \rightarrow \mathrm{AFM}$ are coincidental or not. In the former case (type I) typically found in $\mathrm{BiFeO}_{3}$, a high temperature structural transition $(\mathrm{PE}) \rightarrow \mathrm{FE}$, classified as proper or improper, depending whether the polarization $\boldsymbol{P}$ is the primary order parameter or not, is decoupled from a (PM) $\rightarrow$ AFM transition at lower temperature. The electrical polarization reaches several tens of $\mu \mathrm{C} . \mathrm{cm}^{-2}$ but is not drastically affected by the subsequent magnetic transitions, albeit coupling terms between the electric and magnetic order parameters are present. In the latter case (type II) ferroelectricity appears as an induced effect at one of the magnetic transitions and is classified as improper or pseudoproper depending on the critical behaviour of the polarization and electric susceptibility [2]. For those multiferroics, the value of the electrical polarization is several orders of magnitude lower than for proper ferroelectrics but the strong coupling between magnetic and electric order parameters leads to more pronounced cross-coupling magnetoelectric effects, in particular a strong dependence of $\boldsymbol{P}$ with the magnetic field. In the present lecture, I will focus the discussion on magnetoelectrics and on type II multiferroics, specifically on spin-driven multiferroics. Of course, electrical polarization can be induced by charge-ordering or orbital-ordering rather than magnetic-ordering, but this is outside the scope of this work. This lecture aims to introduce the basic concepts required to perform a symmetry analysis of the magnetic structures in this class of materials, and explain at a phenomenological level the coupling between magnetic and electric properties.

This is an Open Access article distributed under the terms of the Creative Commons Attribution-Noncommercial License 3.0, which permits unrestricted use, distribution, and reproduction in any noncommercial medium, provided the original work is properly cited. 


\section{POLAR AND AXIAL VECTORS, CRYSTAL SYMMETRY AND TIME-REVERSAL SYMMETRY}

Because for magnetoelectric and multiferroics we are dealing with crystalline materials that undergo a magnetic ordering transition and possibly a coincidental ferroelectric transition, the mechanical representations to be considered must take into account the symmetry transformations of polar vectors but also pseudo-vectors. In a classical description, the magnetic moments of the systems are represented by pseudo-vectors (also known as axial vectors) which are directly related to the total angular momentum of a system of unpaired electrons (current loops). Pseudo-vectors transform like polar vectors under proper rotations, but acquire a sign change with respect to polar vectors under improper symmetry operations (inversion, mirrors, and roto-inversions).

In addition to all crystallographic symmetries, one also needs to introduce the time-reversal symmetry operation, which has for effect to reverse the direction of motion of all particles in the system. Here I refer to the "classical" time-reversal symmetry, noted $1^{\prime}$ or $(1 \mid 0,0,0)^{\prime}$ if one uses the Seitz symbol [3], also known as $T$-symmetry and corresponds to a linear operator. We note that this is a different symmetry operator than the time-reversal operation used in Quantum mechanics (Wigner time-reversal) sometimes noted $T^{*}$ or $K$, an anti-linear operator that inverts the direction of time $(t \longrightarrow-t)$ but also compex-conjugates $\left(\psi \longrightarrow \psi^{*}\right)$, a symmetry present when the Hamiltonian of the problem is real. The time-reversal symmetry we will consider here $\left(1^{\prime}\right)$ will have the obvious effect of reversing the direction of a magnetic moment but no influence on polar vectors. The effect of time-reversal symmetry and diverse symmetry operations on polar and axial vectors are represented in Fig. 1. We will see in the following sections that $1^{\prime}$ can appear in the space group symmetry combined with other symmetries or as a separate symmetry operator in the magnetic space group and point group, even in the magnetically ordered state.

\section{MAGNETIC GROUPS}

\subsection{D magnetic space groups}

The 3-dimensional magnetic space group $(M)$ are build by combining the 230 3-dimensional crystallographic space group $(G)$ with time-reversal symmetry. In the terminology of group theory, this corresponds to the direct product of the space groups $G$ with the set $\left\{1,1^{\prime}\right\}, M=G \otimes\left\{1,1^{\prime}\right\}$. Each symmetry operation $(R \mid t)$ of $G$ (in the Seitz notation where $R$ and $t$ are respectively the rotational and translational part of the given symmetry operation) can exist as such i.e. not combined with $1^{\prime}$, and is referred to as a white symmetry operation (or unprimed), or combined with $1^{\prime}$, often noted $(R \mid t)^{\prime}$, and referred to as a black symmetry operation (or primed). There are 1651 such 3D magnetic groups that have been classified by Shubnikov and Heesch [4].

In the paramagnetic state of a system, the absence of a net magnetic moment on all sites (timeaverage quantity is zero) imposes the following:

- all operators of $G$ are valid operators of $M$.

- all operators of $G$ combined with $1^{\prime}$ are valid operators of $M$.

The paramagnetic group contains therefore all operators of $G$ and all operators of $G$ combined with $1^{\prime}$. This is a double group, called a "grey" group since all operators of $G$ appear as white and black operators. In particular, the time-reversal symmetry $1^{\prime}$ is a valid operator of $M$. The full symbol of such a group will be $G 1^{\prime}$. For example the magnetic space group of the $3 \mathrm{D}$ space group Pnma in the paramagnetic phase will be Pnma1'. A "grey" group does not allow magnetic ordering for 3-dimensional group. However, it is extremely important to realize that this statement is only valid in 3 dimensions. As we will see in the following sections, in the case of incommensurate structures which can be described by crystallography in $3+\mathrm{d}$ dimensions, the presence of $1^{\prime}$ in the magnetic space group and magnetic point group is not incompatible with magnetic ordering. 


\section{Contribution of Symmetries in Condensed Matter}

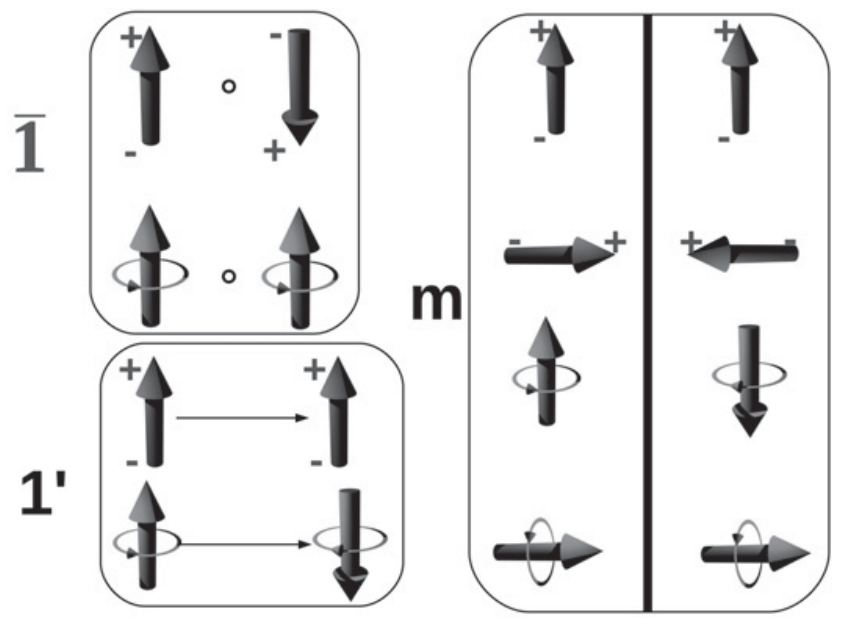

Figure 1. The transformations of a polar vector (signs + and - at the base and tip of the arrow) and axial vector (current loop) under inversion symmetry, time-reversal symmetry and mirror symmetry.

\subsection{Magnetic point groups}

Magnetic point groups, that is the combination of point group symmetry and the symmetry transformation of axial vectors, can be constructed as follows. For each point group $P$ of order n, one can construct a new group of order $2 \mathrm{n}$, say $P^{\prime}$, which contains all symmetry operations of $P$ as such and combined with $1^{\prime}$. This is a grey point group, noted $P 1^{\prime}$. The complete set of magnetic point groups can then be constructed considering $P$ and all isomorphic subgroups of index 2 of $P^{\prime}$. For example, considering the point group $\mathrm{mmm}$. One can construct the double point group $\mathrm{mmm} \mathrm{1}^{\prime}$ that contains all elements of $\mathrm{mmm}$ and the same elements combined with $1^{\prime}$ (a total of 16 point symmetry operations). The isomorphic subgroups of index 2 of $m m m 1^{\prime}$ are $m^{\prime} m^{\prime} m^{\prime}, m^{\prime} m^{\prime} m$ (and corresponding groups by permutations of the axes $m^{\prime} m m^{\prime}, m^{\prime} m^{\prime}$ ), $m m^{\prime}$ (and $m^{\prime} m m, m m^{\prime} m$ ), each containing 8 symmetry operations. One can construct 122 magnetic point groups from the 32 crystallographic point groups. They are listed in [5].

Magnetic point groups play a central role when studying physical phenomena related to magnetic properties of matter, as found in magnetoelectrics and multiferroics. In virtue of Neuman's principle, the various macroscopic physical quantities, expressed by tensors, must be invariants with respect to all symmetry operations of the magnetic point group.

In a very descriptive form, we can state that the rotational part $r$ of a symmetry operator $(r \mid t)$ of the paramagnetic group belongs to the magnetic point group $P$ of the crystal, if the application of $(r \mid t)$ leaves the magnetic structure $M$ invariant, modulo a lattice translation. If applying $(r \mid t)$ changes $M$ into $-M$ (by $-M$ I mean an identical state with all moments pointing in opposite directions), then $r^{\prime}$, belongs to the magnetic point group.

In short notation:

$$
\begin{aligned}
r \in P & \Longleftrightarrow \forall j, \quad(r \mid t) m_{j}=\left(1 \mid t_{0}\right) m_{j} \\
r^{\prime} & \in P \Longleftrightarrow \forall j, \quad(r \mid t)^{\prime} m_{j}=\left(1 \mid t_{0}\right) m_{j} \text { or }(r \mid t) m_{j}=-\left(1 \mid t_{0}\right) m_{j}
\end{aligned}
$$

where $t_{0}$ is a lattice translation. Both $r$ and $r^{\prime}$ are valid operators in the case of grey point groups.

Is is often the very determination of point-group symmetries that represents a real challenge, in particular when microscopic physical quantities such as electric charge or the expectation value of spin 


\section{EPJ Web of Conferences}

form an inhomogeneous state in the crystal. The following section expands the definition given here to the case of modulated structures.

\subsection{Magnetic point groups for inhomogeneous structures}

Most of the recent work on spin-driven ferroelectrics involves complex magnetic structures, often incommensurate with the crystal lattice. These structures can be described by several formalisms. One can extend the superspace formalism widely used for $3+\mathrm{d}$ structural modulations to include magnetism, and employ the magnetic superspace groups whose application have been recently reviewed by J. M. Perez-Mato [6]. In physics, it is more common to use the formalism of wave-vector, and explore the symmetry of the system by explicitly providing the symmetry of the irreducible representations involved in the magnetic order. It is also the formalism of choice for phenomenological theories that require the construction of energy invariants depending on various order parameters (magnetic, structural, polar modes... ). I will use the formalism of propagation vector in this lecture.

\subsubsection{Description of magnetic structures with the formalism of propagation vector}

The easiest way to describe any spatial modulation of ordered moments in a periodic crystal is to expand the magnetic moments in Fourier space. For a simple modulation propagating in the crystal with a wavevector $\vec{k}$, the magnetic moment of an atom $j$ in the unit-cell $\vec{R}_{L}$ with respect to the origin is written:

$$
\vec{m}_{j}\left(\vec{R}_{L}\right)=\vec{S}_{\vec{k}, j} \cdot e^{-i \vec{k} \cdot \vec{R}_{L}}+\vec{S}_{-\vec{k}, j} \cdot e^{i \vec{k} \cdot \vec{R}_{L}}
$$

$\vec{S}_{\vec{k}, j}$ is a complex vector, and the condition of reality for $\vec{m}_{j}\left(\vec{R}_{L}\right)$ imposes that $\vec{S}_{-\vec{k}, j}=\vec{S}_{\vec{k}, j} *$, where * denotes complex-conjugation. One could of course expand $\vec{m}_{j}\left(\vec{R}_{L}\right)$ onto a real field, i.e. a decomposition in cosine and sine functions, but as for Bloch waves, it is far more convenient to deal with Fourier terms $\vec{S}_{\vec{k}, j}$ that are eigenvectors of pure translations. On a real field, the equation would become:

$$
\vec{m}_{j}\left(\vec{R}_{L}\right)=\vec{A}_{\vec{k}, j} \cdot \cos \left(\vec{k} \vec{R}_{L}\right)+\vec{B}_{\vec{k}, j} \cdot \sin \left(\vec{k} \vec{R}_{L}\right)
$$

with $\vec{A}_{\vec{k}, j}=2 \Re\left(\vec{S}_{\vec{k}, j}\right)$ and $\vec{B}_{\vec{k}, j}=2 \Im\left(\vec{S}_{\vec{k}, j}\right)$. The most general modulation for a structure with a single $k$-vector is an ellipse and one can always rewrite the previous equation:

$$
\vec{m}_{j}\left(\vec{R}_{L}\right)=\vec{A}_{\vec{k}, j} \cdot \cos \left(\vec{k} \vec{R}_{L}+\Phi\right)+\vec{B}_{\vec{k}^{\prime}, j}^{\prime} \cdot \sin \left(\vec{k} \vec{R}_{L}+\Phi\right)
$$

where $\vec{B}^{\prime} \vec{k}_{, j}$ is perpendicular to $\vec{A}_{\vec{k}, j}$ and in the $\vec{A}_{\vec{k}, j}, \vec{B}_{\vec{k}, j}$ plane and $\Phi$ is a phase. The formalism of eq. (3.2) can be extended to include more than one $k$-vector, for example required in the case of conical structures (one $k$ inside the Brillouin zone and $k=0$ ). There are of course some further simplifications in the case of $k=0$ or if $k$ is at the surface of the Brillouin zone, for which only the first term in eq. (3.2) is required and $\vec{S}_{\vec{k}, j}$ is a real vector.

\subsubsection{Point-group for modulated structures with $\mathrm{k}$ inside the Brillouin zone}

For modulated structures, the concept of point symmetry is more difficult to grasp because one must determine the macroscopic invariance of physical quantities that are related to objects spatially modulated at the atomic scale. A very good discussion on the subject has been given by Dvorák and co-workers [7]. Essentially, the definition given earlier in eq. (3.2) is still valid but needs to be applied on the different components of the standing waves that describe the magnetically ordered state: The rotational part of a symmetry operator is a valid operator of the point group if and only if the only effect of applying the latter results in de-phasing the initial waves by $\vec{k} \vec{t}_{0}$ where $\vec{t}_{0}$ is a direct-lattice vector. Equation (3.2) needs rewriting in the context of the Fourier expansion (eq. (3.2)). Importantly, in the complex field of $\left(\vec{S}_{\vec{k}, j}, \vec{S}_{-\vec{k}, j}\right)$, a symmetry operation that transforms $\vec{S}_{\vec{k}, j}$ into $\vec{S}_{-\vec{k}, j}$ modulo a translation 


\section{Contribution of Symmetries in Condensed Matter}

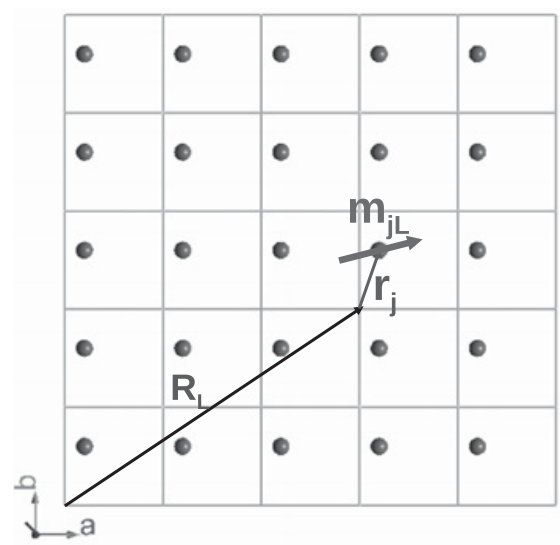

Figure 2. Magnetic moment of atom $j$, at position $\vec{r}_{j}$ in the primitive unit-cell, and global position $\vec{R}_{j L}=\vec{r}_{j}+\vec{R}_{L}$ with respect to the origin, where $\vec{R}_{L}$ is a direct lattice vector.

$-t_{0}$ will also be a valid operation of the point group:

$$
r \in P \Longleftrightarrow \forall j, \quad(r \mid t) \vec{S}_{\vec{k}, j}=\vec{S}_{\vec{k}, j} e^{i \vec{k} \vec{t}_{0}} \text { or }(r \mid t) \vec{S}_{\vec{k}, j}=\vec{S}_{-\vec{k}, j} e^{-i \vec{k} \vec{k}_{0}}
$$

It is clear from the previous definition that the magnetic point symmetry of the crystal can not be fully captured from the symmetry of the little group (also known as the propagation group, the group that leaves the wave-vector invariant), since a symmetry operation that changes $k$ into $-k$ can potentially leave the magnetic configuration invariant. For a single- $k$ structure, an adequate symmetry analysis will need to include, at least, the symmetry elements of the little group and those that exchange $k$ and $-k$.

\subsubsection{Time-reversal symmetry}

The effect of time-reversal symmetry must be considered carefully when studying magnetic structures with $k$ inside the Brillouin zone. Let consider an incommensurate magnetic structure with a single- $k$, as shown in Fig. 3. Because of the incommensurability, for an infinite crystal, all values of the sinusoidal function are realised somewhere in the crystal at a lattice node, meaning that one can always find a lattice translation $\vec{T}_{L}$ such that $\vec{k} \vec{T}_{L}=\pi$. Applying time-reversal followed by a translation by $\vec{T}_{L}$ leaves the magnetic structure invariant. Therefore time-reversal symmetry is a valid operator of the point group. The same applies for a square modulation made of odd-harmonics of the wave vector $k, 3 k, 5 k, \ldots$ since $e^{i \vec{k} \cdot \vec{T}_{L}}=e^{3 i \vec{k} \cdot \vec{T}_{L}}=e^{i s \vec{k} \cdot \vec{T}_{L}}=-1$. However, time-reversal is not necessarily a valid operator for a magnetic structure with $k$ inside the Brillouin zone but commensurate. A typical example would be a structure described by the wave-vector $\frac{1}{3}$ in a given direction, say $z$. Lattice translations can only rephase the wave by $2 \pi / 3$ or $4 \pi / 3$, which do not leave the structure invariant when combined with $1^{\prime}$ symmetry. The presence or not of time-reversal symmetry has important consequences for the allowed magnetoelectric coupling terms, as explained in the following sections.

\subsubsection{Representation analysis for incommensurate structures}

There are essentially two methods to determine the point symmetry in such cases, the use of corepresentations [8] and using the complete irreducible representations of space groups. The former method has been exposed at length in a recent article [8] which includes several examples of symmetry analysis of multiferroic materials. In this method, one considers the symmetry elements of the little group and the elements whose rotational part change $k$ into $-k$, which are combined with the complex conjugation operator $K$. For example, in a centrosymmetric group with $k$ inside the Brillouin zone, 


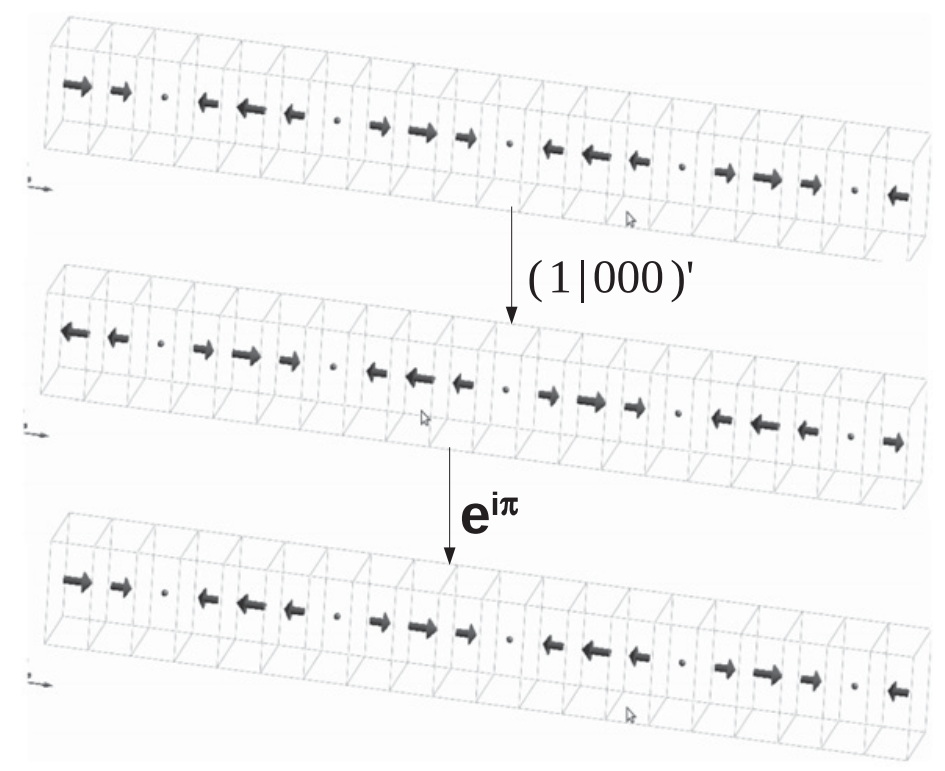

Figure 3. Invariance of a spin-density wave under time-reversal symmetry. The combined effect of time-reversal and a translation $\vec{T}$ such that $\mathrm{e}^{i \vec{k} \vec{T}}=-1$ leaves the magnetic modulation invariant.

the inversion $(\overline{1} \mid 000)$ transforms $k$ into $-k$ and does not belong to the little group. However, the inversion combined with complex conjugation $K(\overline{1} \mid 000)$ is a symmetry operation that leaves $k$ invariant. Co-representations can be constructed easily from Kovalev's tables [9], leading to all necessary symmetry information to reconstruct the magnetic point symmetry.

The second method, which employs the complete irreducible representations of space group is more general. It is completely equivalent to the use of co-representations in the cases where only $k$ and $-k$ belongs to the star of the wave-vector. It is however more complete and can also be used in the most complex symmetry cases for example when dealing with multi- $k$ structures. This is the method I will employ in the following sections and for the examples.

In this method, one considers all symmetry elements of the paramagnetic space group. The matrix representative of each symmetry operation of a given irreducible representation include blocks relative to the full star of the propagation vector. For example, for an incommensurate structure with only $\{k,-k\}$ in the star, a one-dimensional representation in the little group, will be two-dimensional in the complete group (on the basis of $\left(\vec{S}_{\vec{k}, j}, \vec{S}_{-\vec{k}, j}\right)$ ). For a trigonal group $P \overline{3}$ and a wave-vector $\vec{k}=2 \pi(\delta, \delta, 0)$, a one-dimensional irreducible representation in the little-group will be six-dimensional in the full representation since the star is made of $\left\{\vec{k},-\vec{k}, \vec{k}_{2},-\vec{k}_{2}, \vec{k}_{3},-\vec{k}_{3}\right\}$ with $\vec{k}_{2}=2 \pi(\delta,-2 \delta, 0)$ and $\vec{k}_{3}=2 \pi(-2 \delta, \delta, 0)$. The full point symmetry is obviously captured by the analysis in the full group, including the relation between orientation-domains and $k$-domains since the symmetry elements contained in the space group but absent in the little group, mix the matrix blocks related to different $k$-vectors of the star.

\section{REPRESENTATION ANALYSIS FOR MAGNETOELECTRICS AND MULTIFERROICS}

\subsection{Classification of ferroic states under parity and time-symmetry}

The three ferroic states, ferroelectricity, ferromagnetism and ferroelasticity can be classified according to their symmetry transformations under parity(inversion) and time-reversal symmetry. Ferromagnetism is parity-even and time-odd, ferroelectricity is parity-odd and time-even, and ferroelasticity is 


\section{Contribution of Symmetries in Condensed Matter}

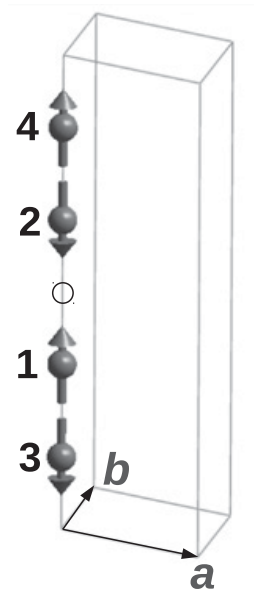

Figure 4. Zero-field magnetic structure of $\mathrm{Cr}_{2} \mathrm{O}_{3}$. For clarity, only $\mathrm{Cr}$ ions along the $0,0, z$ line are shown. Inversion symmetry is displayed as an open circle.

parity-even and time-even. The fourth ferroic state of ferrotoroidicity has recently been proposed alongside the aforementioned ferroics, odd under both parity and time. However, there are no experimental proofs that ferrotoroidicity can act as the primary order-parameter of the phase transition, and has been always observed so far as a secondary order parameter coupled to the primary magnetic ordering.

\subsection{Linear magnetoelectrics}

The linear magnetoelectric effect can only appear in the magnetically ordered state of crystals of specific symmetry [10]. The thermodynamic potential can be expended in mixed polynomials of the electric field $\vec{E}$ and magnetic field components $\vec{H}$ :

$$
F(\vec{E}, \vec{H})=F_{0}-\alpha_{i j} E_{i} H_{j}-\frac{1}{2} \beta_{i j k} E_{i} H_{j} H_{k}-\frac{1}{2} \gamma_{i j k} E_{i} E_{j} H_{k}+\ldots
$$

from which the dependence of the electric polarization and magnetization can be derived by differentiation of $F$ with respect to their conjugate fields.

Since $\vec{E}$ is time-even and $\vec{H}$ is time-odd, the linear magnetoelectric effect is forbidden in the paramagnetic state. Likewise linear magnetoelectric effects can not originate from an incommensurate structure that preserves time-reversal symmetry in the point group as seen in the previous section. Furthermore, for centrosymmetric crystals, the linear magnetoelectric effect can be observed only if inversion symmetry is primed (time-reversed), which leaves invariant the product $E H$. The list of magnetic point groups and their compatibility with magnetoelectric invariants up to the third order are given in [5].

\subsubsection{Example: $\mathrm{Cr}_{2} \mathrm{O}_{3}$}

Let illustrate the symmetry analysis in the case of the prototypal linear magnetoelectric $\mathrm{Cr}_{2} \mathrm{O}_{3}$. The linear magnetoelectric effect was predicted in this material by Dzyalonshinskii [11] and first measured by Astrov [12] and Folen [13]. $\mathrm{Cr}_{2} \mathrm{O}_{3}$ crystallizes in the space group $R \overline{3} c 1^{\prime}$. Cr ions occupy the $12 \mathrm{c}$ Wyckoff site (point symmetry 3), with position 0,0,0.3476. Magnetic order sets in at $307 \mathrm{~K}$ with a wavevector $k=0$. The magnetic structure, shown in fig. 4 in the hexagonal setting of the group, corresponds to an antiferromagnetic arrangement with the moments aligned along the $c$-axis that transforms as a 
Table 1. Irreducible representation corresponding to the zero-field magnetic structure of $\mathrm{Cr}_{2} \mathrm{O}_{3}$, space group $R \overline{3} c 1^{\prime}$. The transformation properties of the differrent components of the electric and magnetic field under symmetries of the paramagnetic group are also shown (see text for details).

\begin{tabular}{|c|c|c|c|c|c|c|c|}
\hline & $(1 \mid 0,0,0)$ & $(3 \mid 0,0,0)$ & $\left(2 \mid 0,0, \frac{1}{2}\right)$ & $(\overline{1} \mid 0,0,0)$ & $(-3 \mid 0,0,0)$ & $\left(m \mid 0,0, \frac{1}{2}\right)$ & $1^{\prime}$ \\
\hline$\Gamma_{2}$ & 1 & 1 & 1 & -1 & -1 & -1 & -1 \\
\hline$E_{z}$ & $E_{z}$ & $E_{z}$ & $-E_{z}$ & $-E_{z}$ & $-E_{z}$ & $E_{z}$ & $E_{z}$ \\
\hline$H_{z}$ & $H_{z}$ & $H_{z}$ & $-H_{z}$ & $H_{z}$ & $H_{z}$ & $-H_{z}$ & $-H_{z}$ \\
\hline$E_{x^{\prime}}$ & $E_{x^{\prime}}$ & $-\frac{1}{2} E_{x^{\prime}}+\frac{\sqrt{3}}{2} E_{y^{\prime}}$ & $E_{x^{\prime}}$ & $-E_{x^{\prime}}$ & $\frac{1}{2} E_{x^{\prime}}-\frac{\sqrt{3}}{2} E_{y^{\prime}}$ & $-E_{x^{\prime}}$ & $E_{x^{\prime}}$ \\
\hline$H_{x^{\prime}}$ & $H_{x^{\prime}}$ & $-\frac{1}{2} H_{x^{\prime}}+\frac{\sqrt{3}}{2} H_{y^{\prime}}$ & $H_{x^{\prime}}$ & $H_{x^{\prime}}$ & $-\frac{1}{2} H_{x^{\prime}}+\frac{\sqrt{3}}{2} H_{y^{\prime}}$ & $H_{x^{\prime}}$ & $-H_{x^{\prime}}$ \\
\hline$E_{y^{\prime}}$ & $E_{y^{\prime}}$ & $-\frac{\sqrt{3}}{2} E_{x^{\prime}}-\frac{1}{2} E_{y^{\prime}}$ & $-E_{y^{\prime}}$ & $-E_{y^{\prime}}$ & $\frac{\sqrt{3}}{2} E_{x^{\prime}}+\frac{1}{2} E_{y^{\prime}}$ & $E_{y^{\prime}}$ & $E_{y^{\prime}}$ \\
\hline$H_{y^{\prime}}$ & $H_{y^{\prime}}$ & $-\frac{\sqrt{3}}{2} H_{x^{\prime}}-\frac{1}{2} H_{y^{\prime}}$ & $-H_{y^{\prime}}$ & $H_{y^{\prime}}$ & $-\frac{\sqrt{3}}{2} H_{x^{\prime}}-\frac{1}{2} H_{y^{\prime}}$ & $-H_{y^{\prime}}$ & $-H_{y^{\prime}}$ \\
\hline
\end{tabular}

single irreducible representation (irrep.) of the group $\left(\Gamma_{2}\right)$. The matrices representative of this irrep. are listed in Table 1. Obviously time-reversal symmetry is lost in point group since $k=0$. It is also clear that the magnetic structure is invariant by three-fold rotation and two-fold rotations (a two-fold rotation permutes atom 1 and 2 on one hand and 3 and 4 on the other). However the magnetic structure is flipped by spatial inversion (shown as an open circle) and mirror symmetry (c glide-plane), so that $\overline{3}^{\prime}, \overline{1}^{\prime}$ and $c^{\prime}$ are valid operators. The magnetic point-group is therefore $\overline{3}^{\prime} m^{\prime}$, which allows linear magnetoelectric coupling of the form $E_{z} H_{z}$. The transformation properties of $E_{z}$ and $H_{z}$ under the symmetry operations of the paramagnetic group are also displayed in Table 1 . It is easy to verify that the quantity $\rho E_{z} H_{z}$, where $\rho$ is the magnetic order parameter transforming as $\Gamma_{2}$, is invariant under all operations of the group (including time-reversal symmetry), and therefore is an allowed invariant in the thermodynamic potential. Taking the $x^{\prime}$-direction perpendicular to the $x, 2 x, z$ mirror and $y^{\prime}$ perpendicular to $x^{\prime}$ and $z$, one can write also the transformation properties of $E_{x^{\prime}}, E_{y^{\prime}}, H_{x^{\prime}}$ and $H_{y^{\prime}}$. The transformations are shown in Table 1 . One can verify that the quantity $\rho\left(E_{x^{\prime}} H_{x^{\prime}}+E_{y^{\prime}} H_{y^{\prime}}\right)$ is invariant, i.e. a linear magnetoelectric coupling of equal magnitude is allowed in the $x^{\prime}$ and $y^{\prime}$ directions.

\subsection{Study of spin-driven ferroelectrics}

In spin-driven ferroelectrics, ferroelectricity originates from the symmetry lowering associated with magnetic ordering, i.e. the magnetic point group must be polar. In the Landau theory of phase transition, for such materials we refer to the magnetic ordering as the primary order parameter, and ferroelectricity (or the polar modes) as the secondary order parameter. One can also say that these are proper antiferromagnets and improper ferroelectrics. The following examples illustrate the use of representation analysis, the first one for a commensurate spin-driven ferroelectric and the second one in the case of an incommensurate magnetic structure.

\subsubsection{Study of the commensurate magnet $\mathrm{Ca}_{3} \mathrm{CoMnO}_{6}$}

$\mathrm{Ca}_{3} \mathrm{CoMnO}_{6}$ is a quasi one-dimensional magnet analog to $\mathrm{Ca}_{3} \mathrm{Co}_{2} \mathrm{O}_{6}$, famously known for displaying magnetization steps below its Neel temperature [14]. $\mathrm{Ca}_{3} \mathrm{CoMnO}_{6}$ crystallizes in the $R \overline{3} c$ space group, and the $\mathrm{Mn}^{4+}$ and $\mathrm{Co}^{2+}$ ions are ordered within chains running along the $c$-axis of the crystal structure. $\mathrm{Mn}^{4+}$ occupy the Wickoff site $6 \mathrm{~b}$ at position $0,0,0$ (point symmetry $\overline{3}$ ) and $\mathrm{Co}^{2+}$ occupy the Wickoff site $6 \mathrm{a}$ at position $0,0,1 / 4$ (point symmetry 32 ). The compound develops long-range magnetic ordering below $16.5 \mathrm{~K}$, temperature below which the system becomes ferroelectric [15]. The magnetic ordering is characterized by the wave-vector $k=0$, and time-reversal symmetry is lost in the point group. A model for the magnetic structure proposed from powder neutron diffraction experiment is shown 


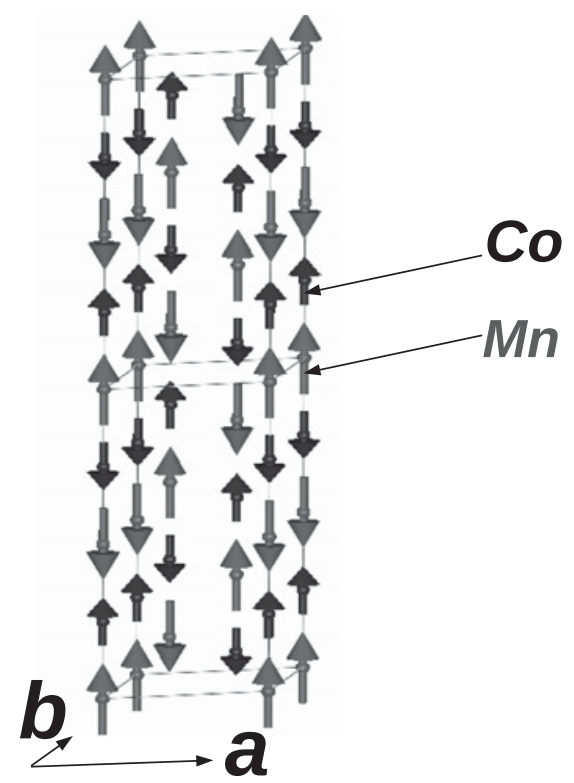

Figure 5. Zero-field magnetic structure of $\mathrm{Ca}_{3} \mathrm{MnCoO}_{6}$. The $\mathrm{Mn}$ and $\mathrm{Co}$ ions are shown as light-grey and black spheres respectively. The structure is shown in the hexagonal setting of the rhombohedral group $R \overline{3} c$.

Table 2. Irreducible representations involve in the zero-field magnetic structure of $\mathrm{Ca}_{3} \mathrm{CoMnO}_{6}$, space group $R \overline{3} c 1^{\prime}$. The transformation properties of the $z$-component of the electric polarization under symmetries of the paramagnetic group is also shown (see text for details).

\begin{tabular}{|c|c|c|c|c|c|c|c|}
\hline & $(1 \mid 0,0,0)$ & $(3 \mid 0,0,0)$ & $\left(2 \mid 0,0, \frac{1}{2}\right)$ & $(\overline{1} \mid 0,0,0)$ & $(-3 \mid 0,0,0)$ & $\left(m \mid 0,0, \frac{1}{2}\right)$ & $1^{\prime}$ \\
\hline$\Gamma_{1}$ & 1 & 1 & 1 & 1 & 1 & 1 & -1 \\
\hline$\Gamma_{4}$ & 1 & 1 & -1 & -1 & -1 & 1 & -1 \\
\hline $\mathrm{P}_{z}$ & $\mathrm{P}_{z}$ & $\mathrm{P}_{z}$ & $-\mathrm{P}_{z}$ & $-\mathrm{P}_{z}$ & $-\mathrm{P}_{z}$ & $\mathrm{P}_{z}$ & $\mathrm{P}_{z}$ \\
\hline
\end{tabular}

in Fig. 5. It appears that each magnetic site $(\mathrm{Mn}, \mathrm{Co})$ orders with a different one-dimensional irrep. of the group. Mn moments order according to the totally symmetric representation of the group. The Co magnetic sublattice is invariant by the three-fold rotation and mirror symmetry ( $c$ glide) but odd under two-fold rotations and inversion. The character of the two irreps. are shown in Table 2. By inspection of the magnetic configuration and table, it is clear that the magnetic point group is $3 m$ since only the symmetry operations that have the same character for the two irreps are preserved in the magnetic group. The system must be ferroelectric along $c$, as indeed measured in the study in [15]. If one labels the magnetic order parameter of the Mn and Co sublattices $\rho_{1}$ and $\rho_{2}$ respectively, it is easy to verify from Table 2 that the trilinear coupling term $\rho_{1} \rho_{2} P_{z}$ is indeed invariant under all symmetry operations of the paramagnetic group.

\subsubsection{Study of the incommensurate spin-driven ferroelectric $\mathrm{TbMnO}_{3}$}

$\mathrm{TbMnO}_{3}$ has been the first example of improper (or pseudo-proper[2]) ferroelectricity induced by a cycloidal magnetic ordering [16]. A model for the magnetic structure was proposed by M. Kenzelmann [17] from single-crystal neutron diffraction data. $\mathrm{TbMnO}_{3}$ crystallizes in the Pbnm space group, and the Mn occupy the $4 \mathrm{~b}$ site at position 1/2,0,0. There are four equivalent $\mathrm{Mn}$ atoms in the unit-cell, labelled as follows: Mn1: 1/2,0,0, Mn2: 0,1/2,1/2, Mn3: 1/2,0,1/2 and Mn4: 0,1/2,0. Tb ions also bear a magnetic moment, but for simplicity, we will consider only the magnetic moments on the Mn ions. Incommensurate magnetic order sets in at $41 \mathrm{~K}$ with the propagation vector $k=2 \pi(0, \delta, 0)$ and $\delta \sim$ 0.276. Symmetry analysis in the little group show that the magnetic representation $\tau$ for the Mn site can 


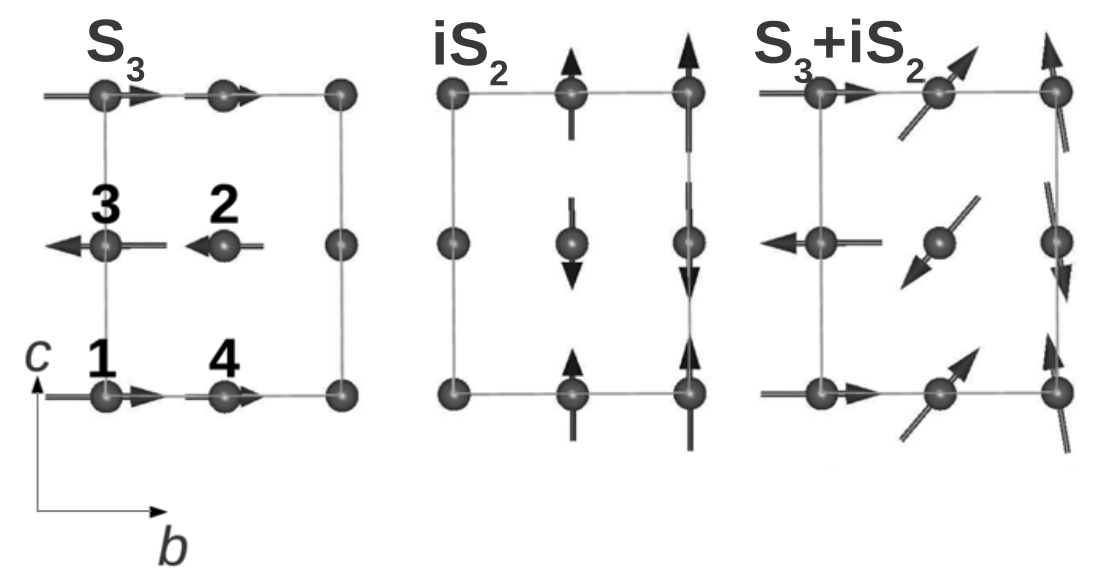

Figure 6. Zero-field magnetic structure of $\mathrm{TbMnO}_{3}$. The two magnetic modes $S_{3}$ and $i S_{2}$ (see text for details) are shown decomposed as well as combined to form the low-temperature cycloidal structure.

Table 3. Irreducible representations of the little group of space group $P b n m 1^{\prime}$ with wave-vector $k=2 \pi(0, \delta, 0)$. $\epsilon=e^{-i \pi \delta}$.

\begin{tabular}{|c|c|c|c|c|}
\hline & $(1 \mid 0,0,0)$ & $\left(2_{y} \mid \frac{1}{2}, \frac{1}{2}, \frac{1}{2}\right)$ & $\left(m_{x y} \mid 0,0, \frac{1}{2}\right)$ & $\left(m_{y z} \mid \frac{1}{2}, \frac{1}{2}, 0\right)$ \\
\hline$\tau_{1}$ & 1 & $\epsilon$ & 1 & $\epsilon$ \\
\hline$\tau_{2}$ & 1 & $\epsilon$ & -1 & $-\epsilon$ \\
\hline$\tau_{3}$ & 1 & $-\epsilon$ & 1 & $-\epsilon$ \\
\hline$\tau_{4}$ & 1 & $-\epsilon$ & -1 & $\epsilon$ \\
\hline
\end{tabular}

be decomposed into the four one-dimensional irreducible representations: $\tau=3 \tau_{1}+3 \tau_{2}+3 \tau_{3}+3 \tau_{4}$ (following Kovalev's notation [9]). The character of the representations are given in Table 3 and $\epsilon=e^{i \pi \delta}$. There are in fact two magnetic transitions at $\mathrm{T}_{N 1}=41 \mathrm{~K}$ and $\mathrm{T}_{N 2}=28 \mathrm{~K}$. The first one corresponds to a longitudinal amplitude-modulated structure, consistent with the $\tau_{3}$ representation and the moments along the $b$-axis. The corresponding magnetic mode $\vec{S}_{3}$ is:

$$
\vec{S}_{3}=M_{b}\left(\vec{S}_{1 b}-\epsilon \vec{S}_{2 b}-\vec{S}_{3 b}+\epsilon \vec{S}_{4 b}\right)
$$

where $M_{b}$ is a real amplitude and the $S_{i b}$ are real-unit vectors along $b$ for each $\operatorname{Mn}$ site $(i=1,4)$.

At the second transition, an additional mode develops in phase quadrature with the first one, giving rise to a cycloidal modulation (i.e. the moments rotate in a plane that contains the propagation vector). This second mode has $\tau_{2}$ symmetry and can be written:

$$
\vec{S}_{2}=M_{c}\left(\vec{S}_{1 c}-\epsilon \vec{S}_{2 c}-\vec{S}_{3 c}+\epsilon \vec{S}_{4 c}\right)
$$

The magnetic structure below $28 \mathrm{~K}$, taking into account the phase quadrature between $\vec{S}_{3}$ and $\vec{S}_{2}$ corresponds to a mode $\vec{S}=\vec{S}_{3}+i \vec{S}_{2}$. The magnetic structures above and below $28 \mathrm{~K}$ are represented in Fig. 6. The full magnetic symmetry can not be captured from the symmetry operations of the little group itself and one needs to use the co-reps (this example is treated in [8]) or the complete irreducible representations $\Delta_{2}$ and $\Delta_{3}$ of the space group Pbnm, built from the irreducible representations of the little group $\tau_{2}$ and $\tau_{3}$, as shown in Table 4. Here, I remind that these matrices act in the vector space formed by $\left(S_{2}, S_{2}^{*}\right)$ and $\left(S_{3}, S_{3}^{*}\right)$.

Any of the two modes taken separately $\left(S_{3}\right.$ or $\left.i S_{2}\right)$ are invariant under all operations of the group, since applying the different symmetries only add a phase to each of these modes. So the magnetic point symmetry of the high-temperature phase of $\mathrm{TbMnO}_{3}$ is $m m m 1^{\prime}$, non-ferroelectric. In contrast for the 


\section{Contribution of Symmetries in Condensed Matter}

Table 4. Irreducible co-representations involved in the magnetically ordered phases of $\mathrm{TbMnO}_{3}$, space group $P b n m 1^{\prime}$ with wave-vector $k=2 \pi(0, \delta, 0) . \epsilon=e^{-i \pi \delta}$.

\begin{tabular}{|c|c|c|c|c|c|}
\hline Sym. op. & $(1 \mid 0,0,0)$ & $\left(2_{y} \mid \frac{1}{2}, \frac{1}{2}, \frac{1}{2}\right)$ & $\left(m_{x y} \mid 0,0, \frac{1}{2}\right)$ & $\left(m_{y z} \mid \frac{1}{2}, \frac{1}{2}, 0\right)$ & $(\overline{1} \mid 0,0,0)$ \\
\hline$\Delta_{2}$ & $\left(\begin{array}{ll}1 & 0 \\
0 & 1\end{array}\right)$ & $\left(\begin{array}{cc}\epsilon & 0 \\
0 & \epsilon^{*}\end{array}\right)$ & $\left(\begin{array}{cc}-1 & 0 \\
0 & -1\end{array}\right)$ & $\left(\begin{array}{cc}-\epsilon & 0 \\
0 & -\epsilon^{*}\end{array}\right)$ & $\left(\begin{array}{ll}0 & 1 \\
1 & 0\end{array}\right)$ \\
\hline$\Delta_{3}$ & $\left(\begin{array}{ll}1 & 0 \\
0 & 1\end{array}\right)$ & $\left(\begin{array}{cc}-\epsilon & 0 \\
0 & -\epsilon^{*}\end{array}\right)$ & $\left(\begin{array}{ll}1 & 0 \\
0 & 1\end{array}\right)$ & $\left(\begin{array}{cc}-\epsilon & 0 \\
0 & -\epsilon^{*}\end{array}\right)$ & $\left(\begin{array}{ll}0 & 1 \\
1 & 0\end{array}\right)$ \\
\hline Sym. op. & $\left(\begin{array}{c}2 x \mid \frac{1}{2}, \frac{1}{2}, 0 \\
)\end{array}\right)$ & $\left(\begin{array}{cc}2_{z} \mid 0,0, \frac{1}{2}\end{array}\right)$ & $\left(m_{x z} \mid \frac{1}{2}, \frac{1}{2}, \frac{1}{2}\right)$ & $1^{\prime}$ & $(1 \mid 0,1,0)$ \\
\hline$\Delta_{2}$ & $\left(\begin{array}{cc}0 & -\epsilon \\
-\epsilon^{*} & 0\end{array}\right)$ & $\left(\begin{array}{cc}0 & -1 \\
-1 & 0\end{array}\right)$ & $\left(\begin{array}{cc}0 & \epsilon \\
\epsilon^{*} & 0\end{array}\right)$ & $\left(\begin{array}{cc}-1 & 0 \\
0 & -1\end{array}\right)$ & $\left(\begin{array}{cc}\epsilon^{2} & 0 \\
0 & \epsilon^{* 2}\end{array}\right)$ \\
\hline$\Delta_{3}$ & $\left(\begin{array}{cc}0 & -\epsilon \\
-\epsilon^{*} & 0\end{array}\right)$ & $\left(\begin{array}{ll}0 & 1 \\
1 & 0\end{array}\right)$ & $\left(\begin{array}{cc}0 & -\epsilon \\
-\epsilon^{*} & 0\end{array}\right)$ & $\left(\begin{array}{cc}-1 & 0 \\
0 & -1\end{array}\right)$ & $\left(\begin{array}{cc}\epsilon^{2} & 0 \\
0 & \epsilon^{* 2}\end{array}\right)$ \\
\hline
\end{tabular}

low temperature phase, a number of symmetry operations do not leave the magnetic structure invariant. Let inspect first the symmetry operations of the little group, omitting the identity: the two-fold axis along $y$ introduces a phase of $-\epsilon$ for the $S_{3}$ mode but $+\epsilon$ for the $i S_{2}$ mode. So $S=S_{3}+i S_{2}$ is not invariant by this symmetry axis. Likewise, the mirror $m_{x y}$ is lost. However, the $m_{y z}$ mirror is preserved. Let now focus on the other symmetry operations. The matrices representatives for the inversion are identical for $\Delta_{3}$ and $\Delta_{2}$, but off-diagonal. This means that this symmetry operation transform $S_{2}$ and $S_{3}$ into their respective complex conjugates. Since $S_{3}$ is real, and $i S_{2}$ purely complex, the inversion transforms $S_{3}+i S_{2}$ into $S_{3}-i S_{2}$, and is therefore lost. Likewise, the two-fold axis along $x$ is lost. However, the two-fold axis along $z$ and the $m_{x z}$ mirror are preserved. The point symmetry in the low-temperature phase is therefore $m m 21^{\prime}$, and the system is ferroelectric along $c$, as observed experimentally. It is also easy to verify from the transformation property of $\boldsymbol{P}$, that the quantity $\left(S_{2} S_{3}^{*}+S_{3}^{*} S_{2}\right) P_{z}$ that expresses the trilinear magnetoelectric coupling between the two magnetic order parameters and $P_{z}$, is invariant. A complete symmetry analysis and treatment using the Landau theory of phase transitions can be found in $[2,18]$.

\section{CONCLUSION}

Representation analysis is an extremely powerful tool to study symmetry-breaking transitions in periodic solids and should be systematically employed to understand phenomenogically the coupling between magnetic order and ferroelectricity in magnetoelectrics and multiferroics. The application of the technique to incommensurate structures is relatively straightforward, as shown in the present paper, even for quite complex magnetic structures. The use of representation analysis is now also facilitated by computer programs such as Isotropy [19] or the suite of applications in the Bilbao Crystallographic server [20], including the generation of co-representations of the crystallographic groups.

\section{References}

[1] H. Schmid, Ferroelectrics 162, p. 317, 1994.

[2] P. Tolédano, "Pseudo-proper ferroelectricity and magnetoelectric effects in $\mathrm{TbMnO}_{3}$," Phys. Rev. B 79, p. 094416, 2009.

[3] F. Seitz, Z. Kristallogr. 88, p. 433, 1960.

[4] A. Belov and N. Shubnikov, Color symmetry, Pergamon Press, 1964. 
[5] H. Grimmer, "The periodic system of Shubnikov point groups," Acta Crystallographica Section A 36 (6), pp. 839-845, 1980.

[6] J. Perez-Mato, J. L. Ribeiro, V. Petricek, and M. Aroyo, arXiv:1107.2358, 2011.

[7] V. Dvorak, J. Janovec, and Y. Ishibashi, "Symmetry of Macroscopic Properties of Incommensurate Structures," Journal of the Physical Society of Japan 52, p. 2053, 1983.

[8] P. G. Radaelli and L. C. Chapon, "Symmetry constraints on the electrical polarization in multiferroic materials," Phys. Rev. B 76, p. 054428, 2007.

[9] O. Kovalev, Representations of the Crystallographic Space Groups, Second Edition, Gordon and Breach Science Publishers, 1993.

[10] L. D. Landau and E. M. Lifshitz, Electrodynamics of Continuous Media, Addison-Wesley, 1960.

[11] I. E. Dzyaloshinskii, Sov. Phys JETP 10, p. 628, 1960.

[12] D. Astrov, Sov. Phys JETP 13, p. 729, 1960.

[13] V. J. Folen, G. T. Rado, and E. W. Stalder, Phys. Rev. Lett. 6, p. 607, 1961.

[14] H. Kageyama, K. Yoshimura, K. Kosuge, H. Mitamura, and T. Goto, "Field-Induced Magnetic Transitions in the One-Dimensional Compound $\mathrm{Ca}_{3} \mathrm{Co}_{2} \mathrm{O}_{6}$," Journal of the Physical Society of Japan 66 (6), pp. 1607-1610, 1997.

[15] Y. J. Choi, H. T. Yi, S. Lee, Q. Huang, V. Kiryukhin, and S.-W. Cheong, "Ferroelectricity in an Ising Chain Magnet," Phys. Rev. Lett. 100, p. 047601, 2008.

[16] T. Kimura, T. Goto, H. Shintani, K. Ishizaka, T. Arima, and Y. Tokura, "Magnetic control of ferroelectric polarization," Nature 426 (6962), pp. 55-58, 2003.

[17] M. Kenzelmann, A. B. Harris, S. Jonas, C. Broholm, J. Schefer, S. B. Kim, C. L. Zhang, S.-W. Cheong, O. P. Vajk, and J. W. Lynn, "Magnetic Inversion Symmetry Breaking and Ferroelectricity in $\mathrm{TbMnO}_{3}$," Phys. Rev. Lett. 95, p. 087206, 2005.

[18] A. B. Harris, "Landau analysis of the symmetry of the magnetic structure and magnetoelectric interaction in multiferroics," Phys. Rev. B 76, p. 054447, 2007.

[19] H. Stokes, D. M. Hatch, and B. J. Campbell, ISOTROPY, stokes.byu.edu/isotropy.html., 2007.

[20] M. I. Aroyo, J. M. Perez-Mato, C. Capillas, E. Kroumova, S. Ivantchev, G. Madariaga, A. Kirov, and H. Wondratschek, "Bilbao Crystallographic Server: I. Databases and crystallographic computing programs," Zeitschrift fr Kristallographie 221, pp. 15-27, 2006. 